\title{
Schellings Wesensbestimmung des Christentums in den Vorlesungen über die Methode des akademischen Studiums
}

\author{
Christian Danz ${ }^{1}$ \\ Evangelisch-Theologische Fakultät \\ Universität Wien \\ Austria
}

\begin{abstract}
Schelling's notion of the essence of Christianity

according to his lectures on scientific methodology

This article focuses on Schelling's lectures on scientific methodology, published in 1802. In these Vorlesungen Schelling developed his theory about the essence of Christianity against the background of a "philosopy of identity". The article consists of three sections which investigate Schelling's understanding of Christianity's historical position in the academia. Theology as the science of Christian religion constitutes the essence of Christianity. The article aims to argue that Schelling's understanding of theology as the object matter of philosophy should be seen in light of his view on Christianity's historical position.
\end{abstract}

\section{EINLEITUNG}

Schellings 1802 an der Jenaer Universität gehaltene und 1803 veröffentlichte Vorlesungen über die Methode des akademischen Studiums wollen dem Studierenden im "Chaos" (SW V, 211) der Wissenschaften eine Orientierung verschaffen. Die Einlösung dieser Aufgabe nimmt bei Schelling die Gestalt einer "Methodenlehre des akademischen Studiums" an, die von der Überzeugung geleitet ist, daß eine solche Methodenlehre "nur aus der wirklichen und wahren Erkenntniß des lebendigen Zusammenhangs aller Wissenschaften hervorgehen könne" (SW V, 213). Mit diesem Programm bezieht sich Schelling nicht nur kritisch auf die wissenschaftstheoretischen

\footnotetext{
${ }^{1}$ Prof Dr Christian Danz is Professor für Systematische Theologie, Evangelisch-Theologische Fakultät, Universität Wien. He is a research associate of Prof Dr Johan Buitendag, Department of Systematic Theology and Ethics, Faculty of Theology, University of Pretoria.
} 
Debatten an der Jenaer Universität, ${ }^{2}$ sondern legt selbst ein ambitioniertes Verfahren zur Begründung der Wissenschaften vor. Die Eigentümlichkeit von Schellings Wissenschaftssystematik ist darin zu sehen, daß sie ihren Ausgang nicht bei dem empirischen Wissen und der mit diesem verbundenen Disjunktion von Begriff und Gegenstand nimmt. Vielmehr soll der Kosmos der Wissenschaften in der "Idee des an sich selbst unbedingten Wissens" (SW V, 215) begründet werden. Wissenschaftssystematik wird damit zur Aufgabe der spekulativen Philosophie. Sie vermag als "Wissenschaft aller Wissenschaften" (SW V, 214) die besonderen Wissenschaften als Darstellungen des Absoluten zu begreifen. In diesem Kosmos der Wissenschaften findet auch die Theologie neben Jurisprudenz und Medizin ihren Ort. Sie wird von Schelling als "die erste und oberste" aller Wissenschaften bestimmt, in der "das Innerste der Philosophie objektivirt ist" (SW V, 283). Die Theologie als "unmittelbare Wissenschaft des absoluten und göttlichen Wesens" stellt den "absoluten Indifferenzpunkt objektiv" (ebd.) dar. Diese wissenschaftssystematische Bestimmung der Theologie beinhaltet einen weitreichenden Anspruch. Es ist ihr darum zu tun, auf dem Hintergrund der nachkantischen Problemlage, die Theologie als Wissenschaft zu begründen.

Bereits ein Jahr später ist Schellings identitätsphilosophische Begründung der Theologie als Wissenschaft von Schleiermacher in der Jenaischen Litteraturzeitung der Kritik unterzogen worden. ${ }^{3}$ Schleiermacher wendet gegenüber Schellings Bestimmung der Theologie nicht nur ein, daß diese die "bestimmte[..] Unterscheidung des Positiven in dieser Wissenschaft

\footnotetext{
${ }^{2}$ Siehe hierzu T v Zantwijk, Ist Anthropologie als Wissenschaft möglich? Der "Mensch" in Schmids "enzyklopädischer Topik" und in Schellings "philosophischer Konstruktion der Wissenschaften", in J Jantzen \& P L Österreich (Hrsg), Schellings philosophische Anthropologie, Stuttgart-Bad Cannstatt 2002, 110-154; P Ziche, Die "eine Wissenschaft der Philosophie" und die "verschiedenen philosophischen Wissenschaften". Wissenschaftssystematik und die Darstellung des Absoluten in Ueber das Verhältnis der Naturphilosophie zur Philosophie überhaupt, in K Vieweg (Hrsg), Gegen das "unphilosophische Unwesen". Das Kritische Journal der Philosophie von Schelling und Hegel, Würzburg 2002, 211-222; Ders, Wissenschaft vom Menschen und Philosophie der Natur. Anthropologie zwischen Physiologie und Naturwissenschaften, in O Breitbach \& P Ziche (Hrsg), Naturwissenschaften um 1800. Wissenschaftskultur in Weimar/Jena, Weimar 2001. Schellings Schriften werden im Folgenden nach der von K F A Schelling besorgten Ausgabe, Sämmtliche Werke Bd. I-XIV, Stuttgart/Augsburg 1856-61, unter Angabe des Bandes und der Seitenzahl zitiert.

${ }^{3}$ F Schleiermacher, Rezension F W J Schelling, Vorlesungen über die Methode des akademischen Studiums, 1803, in Jenaische Litteraturzeitung Bd. I, No. 96.97 (1804), 137151, wiederabgedruckt in W Dilthey (Hrsg), Aus Schleiermacher's Leben, in Briefen. 4. Bd, Berlin 1863, 579-593, hier 583: "Sehr merkwürdig muß es nach einer so unumwundenen Erklärung über das Positive erscheinen, daß in Absicht der Theologie sich bloß darauf berufen wird, es werde allgemein angenommen, sie enthalte etwas positives". Siehe hierzu J Dierken, Das Absolute und die Wissenschaften. Zur Architektonik des Wissens bei Schelling und Schleiermacher, in PhJb 99 (1992), 307-328.
} 
von dem rein historischen und realen"4 gerade nicht, wie beansprucht, geleistet habe, sondern vor allem auch, wie der "Indifferenzpunkt der Gegenstand einer realen Wissenschaft werden" kann. ${ }^{5}$ Für Schleiermacher ist dies ein Indiz für eine grundsätzliche Schwierigkeit, mit der Schellings Grundlegung der Wissenschaften im Absoluten insgesamt behaftet ist. Er erblickt sie in einem unzureichenden Begriff des Absoluten, welche Schelling dadurch zu beheben sucht, daß er zwei Zuordnungsmodelle von Absoluten und Realwissenschaften miteinander kombiniert, die sich gerade nicht verbinden lassen. ${ }^{6}$

Schellings Bestimmung der Theologie als objektiver Darstellung des Indifferenzpunktes stellt eine Implikation der identitätsphilosophischen Fassung des Absoluten dar. Nun ist bekanntlich eben dieser Fassung des Absoluten nicht nur von Schleiermacher widersprochen worden. Die Anfragen beziehen sich sowohl auf den identitätsphilosophischen Begriff des Absoluten ${ }^{7}$ als auch auf die mit diesem Begriff verbundene Deutung des Christentums. ${ }^{8}$ Eine Rekonstruktion von Schellings wissenschaftssystematischer Einordnung und Begründung der Theologie in den Vorlesungen über die Methode des akademischen Studiums wird daher nicht schlecht beraten sein, wenn sie mit einer Rekonstruktion des identitätsphilosophischen Programms selbst einsetzt. Dies scheint mir schon aus dem Grund unumgänglich zu sein, als der für Schellings Identitätsphilosophie signifikante Methodenbegriff schon in der Überschrift der Vorlesung erscheint, die der Theologie sowie dem Christentum gewidmet ist. In dem ersten Abschnitt ist deshalb in bündiger Kürze auf Schellings Verständnis der Methode der

\footnotetext{
${ }^{4}$ F Schleiermacher, Rezension, 583.

${ }^{5}$ F Schleiermacher, Rezension, 584.

${ }^{6}$ Vgl F Schleiermacher, Rezension, 584f. Siehe hierzu J Dierken, Das Absolute und die Wissenschaften, 313.

${ }^{7}$ Zur Diskussion von Schellings identitätsphilosophischem Begriff des Absoluten siehe K Düsing, Die erste Gestalt der Metaphysik des Absoluten bei Schelling (Vorlesung und Schriften 1801-1802), in ders (Hrsg), Schellings und Hegels erste absolute Metaphysik (18011802). Zusammenfassende Vorlesungsnachschriften von I P V Troxler, Köln 1988, 133-156; J Hennigfeld, Einheit und Vielheit als grundlegendes Problem in Schellings Systementwürfen, in AZP 14 (1989), 1-15; J Dierken, Das Absolute und die Wissenschaften, 309-319; M Bachmann, Die paradoxale Struktur des Absoluten in Schellings Identitätssystem, in PhJb 101 (1994), 76-97.

${ }^{8}$ Siehe hierzu E Hirsch, Geschichte der neuern evangelischen Theologie im Zusammenhang mit den allgemeinen Bewegungen des europäischen Denkens, Bd. 4. Gütersloh 1952, 411432; W Becker, Über Schellings Konstruktion des Christentums, in D Henrich \& H Wagner (Hrsg), Subjektivität und Metaphysik. Festschrift für Wolfgang Cramer, Frankfurt/Main 1966, 1-20; H Rosenau, Die Differenz im christologischen Denken Schellings, Frankfurt/Main 1985, 53-58; W Jaeschke, Die Vernunft in der Religion. Studien zur Grundlegung der Religionsphilosophie Hegels, Stuttgart-Bad Cannstatt 1986, 159-170.
} 


\section{Schellings Wesensbestimmung des Christentums}

philosophischen Konstruktion einzugehen. Im Ausgang von diesen methodischen Überlegungen ist dann in einem zweiten Schritt Schellings Deutung des Christentums wenigstens in ihren Grundzügen in den Blick zu nehmen. Diese bildet die Grundlage von Schellings Begründung der Theologie als Wissenschaft, und sie ist orientiert an den Leitbegriffen Darstellung und Repräsentation. Abschließen möchte ich mit Schellings Begriff der Theologie, wie er sich aus den systematischen Voraussetzungen der Identitätsphilosophie ergibt. Die These, die ich in diesen drei Argumentationsgängen begründen möchte, lautet, daß Schelling das Christentum als den geschichtlichen Ort versteht, in dem sich die Vernunft in der ihr eigenen Reflexivität erfaßt. Allein aus diesem Grund kann die Theologie als Wissenschaft von der christlichen Religion die objektive Darstellung der Philosophie sein.

\section{PHILOSOPHIE ALS DARSTELLUNG DES BESONDEREN IM ALLGEMEINEN}

"Die neuere Welt ist allgemein die Welt der Gegensätze, und wenn in der alten, aller einzelnen Regungen ungeachtet, doch im Ganzen das Unendliche mit dem Endlichen unter einer gemeinschaftlichen Hülle vereinigt liegt, so hat der Geist der späteren Zeit zuerst diese Hülle gesprengt und jenes in absoluter Entgegensetzung mit diesem erscheinen lassen" (SW V, 272). Entzweiung und Entgegensetzung sind nach der Diagnose Schellings das Signum der modernen Kultur. Schelling ist jedoch nicht nur der Meinung, daß in der modernen Kultur Besonderes und Allgemeines, Endliches und Absolutes, Ideelles und Reelles in einen Gegensatz getreten sind, sondern vor allem der, daß diesen Gegensätzen letztlich eine Grundstruktur zugrunde liegt, nämlich die Forderung, "das Ich außer dem Absoluten zu halten" (SW V, 109). Diese Entgegensetzung beruht jedoch auf einer undurchschauten Abstraktion. Denn die Setzung des Absoluten außer dem Ich ist nur für ein Ich möglich und fällt somit als nichtgesetzte Setzung in das Ich zurück. ${ }^{9}$ Eben dieser "Cirkel der Reflexion" (SW V, 274) wird der modernen Kultur, deren Bewußtsein in dem Idealismus Fichtes zum paradigmatischen Ausdruck gekommen ist, nicht durchsichtig. Deshalb gelangt die an Gegensätzen orientierte moderne Kultur weder zu einem angemessenen Verständnis der Religion, noch zu einer haltbaren Begründung der Wissenschaften.

Schellings Identitätsphilosophie ist es darum zu tun, diese mit der modernen Kultur verbundenen Entgegensetzungen zu überwinden. Tragender

\footnotetext{
${ }^{9}$ Vorlesungen, SW V, 274: "Als das An-sich mußte es ein absolut-Objektives schlechthin außer dem Ich seyn. Dieß ist undenkbar, indem ja eben dieses außer-dem-Ich-Setzen wieder ein Setzen für das Ich und demnach auch im Ich ist."
} 
Grundgedanke dieses philosophischen Programms ist ein Gedanke des Absoluten, den Schelling als Indifferenzpunkt beschreibt. ${ }^{10}$ Dieses Absolute nennt Schelling in seiner ersten identitätsphilosophischen Schrift Darstellung meines Systems der Philosophie absolute Vernunft und erläutert sie in dem ersten Paragraphen "als totale Indifferenz des Subjektiven und Objektiven" (SW IV, 114). ${ }^{11}$ Mit diesem Begriff der absoluten Vernunft sind drei Aspekte verbunden, aus deren innerem Zusammenhang sich erst das Programm von Schellings sogenannter Identitätsphilosophie ergibt. Diese wiederum stellt den begrifflichen und methodischen Rahmen nicht nur von Schellings Wissenschaftssystematik insgesamt dar, sondern auch von der in diesem Zusammenhang vorgenommenen Begründung der Theologie als Wissenschaft.

Zunächst ist das von Schelling geforderte Verständnis von Vernunft das Resultat einer von dem Philosophen erst vorzunehmenden methodischen Abstraktion.

Diese ist so beschaffen, daß von dem Denkenden zu abstrahieren ist.

Das Denken der Vernunft ist jedem anzumuthen; um sie als absolut zu denken, um also auf den Standpunkt zu gelangen, welchen ich fordere, muß vom Denkenden abstrahirt werden. Dem, welcher diese Abstraktion macht, hört die Vernunft unmittelbar auf etwas Subjektives zu seyn, wie sie von den meisten vorgestellt wird, ja sie kann selbst nicht mehr als etwas Objektives gedacht werden, da ein Objektives oder Gedachtes nur im Gegensatz gegen ein Denkendes möglich wird, von dem hier völlig abstrahirt ist; sie wird also durch jene Abstraktion zu dem wahren An-sich, welches eben in den Indifferenzpunkt des Subjektiven und Objektiven fällt.

(SW IV, 114f)

Durch diese von Schelling ausdrücklich als Abstraktion gekennzeichnete Operation, durch die von dem Denkenden als der Instanz, für welche Objekte sind, abstrahiert werden soll, stellt sich die Vernunft als Indifferenz ein. Mit

\footnotetext{
${ }^{10} \mathrm{VgI}$ Vorlesungen, SW V, 281: "Wird nun das Absolute als dasjenige aufgefaßt, was an sich reine Identität, aber als diese zugleich das nothwendige Wesen der beiden Einheiten ist, so haben wir damit den absoluten Indifferenzpunkt der Form und des Wesens aufgefaßt, denjenigen, von dem alle Wissenschaft und Erkenntniß ausfließt."

${ }^{11}$ Ausführlicher hierzu v Vf, Vernunft und Religion. Überlegungen zu Schellings Christentumsdeutung in seinen Journal-Aufsätzen, in K Vieweg (Hrsg), Gegen das “unphilosophische Unwesen”, Würzburg 2002, 197-209.
} 


\section{Schellings Wesensbestimmung des Christentums}

diesem Vernunftbegriff ist nun ein Verständnis von Philosophie als

Darstellung des Besonderen im Allgemeinen verbunden. ${ }^{12}$

Dem Identitätssystem, welches sich "auf den Standpunkt der

Produktion" (SW IV, 109) stellt, geht es gerade nicht um eine Ableitung oder Deduktion der endliche Dinge aus dem Absoluten. ${ }^{13}$ Dies ist der zweite hier zu nennende Aspekt. Er ist, wie schon der eingangs genannte Einwand von Schleiermacher deutlich machte, nicht unerheblich für die Beurteilung von Schellings Begründung der Theologie als Wissenschaft. Schelling selbst hat es ausdrücklich als ein Mißverständnis seines identitätsphilosophischen Programms bezeichnet, wenn man in ihm eine Ableitung von begrifflicher Bestimmtheit aus Unbestimmtheit sehen wollte.

Wie die Erkenntniß, daß die Philosophie im Absoluten sey, so ist auch das ganze Geschäft und Werk der Philosophie durch das Bisherige so weit ins Licht gesetzt, daß der Irrthum der Vorstellung eingesehen werden kann, welche dieses überhaupt [a)] in ein Ableiten, es sey aus dem Absoluten oder welchem andern Princip sonst, oder [b)] in ein Deduciren der wirklichen, erscheinenden Welt, als solcher, oder der Möglichkeit der Erfahrung setzen"

(SW IV, 396)

Ebenso wenig wie es dem Identitätssystem um eine Ableitung des Besonderen aus dem Absoluten zu tun ist, geht es ihm um einen Übergang vom Besonderen zum Absoluten. Es gehört schon zu den frühesten Einsichten Schellings, daß es von dem Unbedingten zu dem Bedingten keinen Übergang geben kann. Positiv besagt dies dann aber für das von Schelling angewandte methodische Verfahren, daß die Überwindung des Gegensatzes von Endlichem und Unendlichem, Besonderem und Allgemeinem auf eine andere Weise vorgenommen werden muß. Dies führt uns zu dem dritten hier einschlägigen Aspekt von Schellings philosophischem Programm.

Schelling selbst hat das von ihm verfolgte methodische Verfahren mit dem Terminus Konstruktion gekennzeichnet und in der "Lehre von der

\footnotetext{
${ }^{12}$ Darstellung, SW IV, 115: "Der Standpunkt der Philosophie ist der Standpunkt der Vernunft, ihre Erkenntniß ist eine Erkenntniß der Dinge, wie sie an sich, d.h. wie sie in der Vernunft sind."

${ }^{13}$ Als ein Deduktionsprogramm haben schon Fichte und Hegel das Identitätssystem gedeutet und dann konstatiert, daß Schelling aus dem als Indifferenz gefaßten Prinzip die Ableitung und Begründung des Besonderen nicht gelungen sei. Vgl J G Fichte, Kommentar "Zur Darstellung von Schellings Identitätssysteme", GA II/5, 503; G W F Hegel, Phänomenologie des Geistes, GW 9, 17. Dem Urteil von Fichte und Hegel schließt sich auch J Dierken, Das Absolute und die Wissenschaften, 318f, an.
} 
philosophischen Construktion [...] eines der wichtigsten Kapitel in der wissenschaftlichen Philosophie" (SW V, 125) gesehen. ${ }^{14}$ Philosophische Konstruktion heißt für Schelling, und zwar in kritischer Absetzung von Kants Begriff der Konstruktion, Darstellung "des Realen im Idealen, des Besonderen im schlechthin Allgemeinen, der Idee." (SW V, 325) ${ }^{15}$ Konstruktion meint dabei eine solche Handlung, in der Besonderes und Allgemeines "selbstthätig" (SW V, 243) zusammengesetzt werden. Folglich sind die Elemente aller philosophischen Konstruktion das Allgemeine und das Besondere. In der philosophischen Konstruktion ist es allein darum zu tun, den "Streit" zwischen dem Absoluten und dem Besonderen zu lösen. ${ }^{16}$ Die philosophische Konstruktion ist somit ein solches methodisches Verfahren, in dem nicht etwa das Absolute selbst konstruiert wird, sondern das Besondere im Medium der Vernunft. Das Absolute ist der Allgemeinheitshorizont, in den das Besondere durch die philosophische Konstruktion gestellt und gerade dadurch wissenschaftlich erkannt wird. ${ }^{17}$ Besonderes und Allgemeines werden folglich in der philosophischen Konstruktion selbsttätig aufeinander bezogen. Dadurch werden Besonderes und Allgemeines nicht separiert und gegeneinander isoliert, sondern das Besondere wird im Medium des Allgemeinen so konstruiert, daß an diesem das Allgemeine zur Darstellung kommt. Dies ist freilich nur dann möglich, wenn durch die philosophische Konstruktion das Besondere als Repräsentationsverhältnis konstituiert wird. Es repräsentiert dann gleichsam das Allgemeine so, daß beide sowohl in einen Zusammenhang gesetzt als auch unterschieden werden. Schelling nennt diesen Verweisungszusammenhang Idee und ordnet ihr die

\footnotetext{
${ }^{14}$ Schelling hat sein eigenes Verständnis der philosophischen Konstruktion am ausführlichsten in seinem Journal-Aufsatz Ueber die Construktion in der Philosophie, SW V, 125-151, erläutert. Vgl auch ders, Fernere Darstellungen aus dem System der Philosophie, SW IV, 407-411. Zu Schellings Verständnis der philosophischen Konstruktion siehe jetzt $P$ Ziche, Die "eine Wissenschaft der Philosophie" und die "verschiedenen philosophischen Wissenschaften", 217-220; T v Zantwijk, Ist Anthropologie als Wissenschaft möglich?, 134149.

${ }^{15}$ Vgl Vorlesungen, SW V, 252: "Die Darstellung des Allgemeinen und Besonderen in der Einheit, heißt überhaupt Construktion, die von der Demonstration wahrhaft nicht unterschieden ist."

${ }^{16}$ Ueber die Construktion, SW V, 135: "Der Verfasser kennt aber doch, wie man schon hieraus sieht, die Elemente aller Construktion, die Kant völlig unbekannt sind, das Absolute, das an sich uneingeschränkt und schlechthin Eines ist, und das Besondere, welches ein Eingeschränktes und nicht Eines, sondern Vieles ist, ein Streit, der aber nur in der Construktion der Idee und durch produktive Einbildung gelöst werden kann".

${ }^{17}$ Vgl Ueber die Construktion, SW V, 134f.: "Es ist nur Ein Princip der Construktion, Eines, womit construiert wird, in der Mathematik wie in der Philosophie. Dem Geometer ist es die in allen Construktionen gleiche und absolute Einheit des Raums, dem Philosophen die des Absoluten."
} 


\section{Schellings Wesensbestimmung des Christentums}

Anschauung zu. ${ }^{18}$ In dieser sind Allgemeines und Besonderes ebenso verbunden wie auch unterschieden. Der Konstruktionsbegriff bzw. das Verfahren der philosophischen Konstruktion ist also geradezu der Gegenbegriff zu Ableitung und zu Erklären. ${ }^{19}$ Der Philosoph erklärt nicht, sondern er konstruiert.

Nimmt man diese drei hier skizzierten Aspekte zusammen, dann ergibt sich für das Gesamtverständnis der sogenannten Identitätsphilosophie, wie sie in den Vorlesungen über die Methode des akademischen Studiums als methodisch-begriffliche Grundlage der Wissenschaftssystematik dient, daß sie eine Anweisung zur eigenständigen Konstruktion des Wissenschaftskosmos darstellt. Es dürfte jetzt auch deutlich geworden sein, warum Schelling seine Vorlesungen unter den Oberbegriff einer Methodologie gestellt hat und diesen die Aufgabe zugewiesen hat, eine "Methodenlehre des akademischen Studiums" zu sein, welche nur aus "der wirklichen und wahren Erkenntniß des lebendigen Zusammenhangs aller Wissenschaften hervorgehen könne" (SW V, 213). Das Absolute stellt im Horizont der Identitätsphilosophie das Medium dar, in dem der Philosoph das Besondere konstruiert. Durch die Konstruktion kommt das Absolute freilich nur gebrochen zur Darstellung, nämlich dadurch, daß das Besondere das Absolute repräsentiert. Es steht gleichsam für diejenige Unbestimmtheit, die bei jeder Bestimmtheitssetzung mitgesetzt wird. Unbestimmtheit ist daher als die Bedingung der Möglichkeit von Bestimmtheit anzusprechen, die bei jeder weiteren Setzung von Bestimmtheit reproduziert wird. Nach diesen Überlegungen zum methodischen Verfahren von Schellings identitätsphilosophischer Wissenschaftssystematik können wir uns nun Schellings Konstruktion der Theologie als Wissenschaft zuwenden.

\section{DAS CHRISTENTUM ALS GESCHICHTLICHES REFLEXIVWERDEN DER VERNUNFT}

Den Vorlesungen über die Methode des akademischen Studiums geht es um eine Begründung der Theologie als Wissenschaft. Die Wissenschaftlichkeit der Theologie wird von Schelling durch die Philosophie begründet. Die Differenz zwischen der Philosophie und den realen Wissenschaften liegt in

\footnotetext{
${ }^{18}$ Ueber die Construktion, SW V, 135: "Es ist, wie schon gesagt, nur Eines, was construiert wird, nämlich Ideen, und alles Abgeleitete wird nicht als Abgeleitetes, sondern in seiner Idee construiert."

${ }^{19} \mathrm{Vgl}$ Vorlesungen, SW V, 320. Siehe hierzu T v Zantwijk, Ist Anthropologie als Wissenschaft möglich?, $131 \mathrm{ff}$.
} 
dem "historische[n] Element" (SW V, 286)..$^{20}$ So sehr nun für jede besondere Wissenschaft ein historisches Element konstitutiv ist, so sehr soll die Theologie noch eine darüber hinausgehende Beziehung auf die Geschichte haben, die gleichsam zu ihrem Wesen selbst gehört. Die Theologie soll als die "höchste Synthese des philosophischen und historischen Wissens" (ebd.) konstruiert werden. Die Begründung dieses Verständnisses von Theologie als Wissenschaft resultiert allein aus dem methodischen Verfahren von Schellings Identitätsphilosophie. Deshalb hängt die Begründung der Theologie von der Möglichkeit einer historischen Konstruktion des Christentums ab. Sie muß folglich im Mittelpunkt von Schellings Aufmerksamkeit stehen.

Schellings Begründung der Theologie ist an dem methodischen Hauptbegriff seiner Identitätsphilosophie orientiert. Dies hat Konsequenzen sowohl für das Verständnis des Christentums als auch der Theologie. Zu erwarten ist in der Methode des akademischen Studiums keine Erklärung oder Ableitung des Christentums als einer historischen Erscheinung, sondern eine Konstruktion der Idee des Christentums. Nach dem bisher zur Methode der philosophischen Konstruktion Ausgeführten kann es sich hierbei nur um eine Darstellung des geschichtlichen Christentums im Medium des Allgemeinen handeln. Nur so kann das Christentum als besondere und in sich vielschichtige historische Erscheinung wissenschaftlich begriffen werden. Schellings Behauptung ist jedoch nicht nur die, daß das Christentum philosophisch konstruiert werden könne, sondern die weiterreichendere, daß das Christentum eine solche Form von Religion darstellt, in der das Verfahren der Konstruktion den Inhalt der Religion bildet. Erst hieraus resultiert Schellings Behauptung, daß die Theologie "den absoluten Indifferenzpunkt objektiv darstellt" (SW V, 283). Damit verbindet sich die Forderung, die Theologie als die höchste Synthese des historischen und des philosophischen Wissens zu konzipieren. Erst mit der Einlösung dieser Aufgabe ist der Begriff der Theologie im Kosmos der Wissenschaften begründet. Dies sei im Folgenden an der nach Schelling zum Wesen der Theologie selbst gehörenden Beziehung auf die Geschichte erörtert.

Schelling sieht die der Theologie eigentümliche Beziehung auf die Geschichte darin, "daß in dem Christenthum das Universum überhaupt als Geschichte, als moralisches Reich, angeschaut wird, und daß diese

\footnotetext{
${ }^{20} \mathrm{VgI}$ Vorlesungen, SW V, 280: "Nun ist aber allgemein einzusehen, daß das reel[I]-Werden einer Idee in beständigem Fortschritt, so daß zwar nie das Einzelne, aber doch das Ganze ihr angemessen ist, sich als Geschichte ausdrücke. [...] Das wirkliche Wissen, da es successive Offenbarung des Urwissens ist, hat demnach nothwendig eine historische Seite, und inwiefern alle Geschichte auf die Realisierung eines äußern Organismus als Ausdrucks von Ideen geht, hat die Wissenschaft auch das nothwendige Streben, sich eine objektive Erscheinung und äußere Existenz [einen äußeren Organismus] zu geben."
} 
allgemeine Anschauung den Grundcharakter desselben ausmacht." (SW V, 287) Das Christentum ist Anschauung des Universums als Geschichte. ${ }^{21}$ Die Theologie als Wissenschaft der christlichen Religion hat also aus dem Grunde eine Beziehung auf die Geschichte, weil die Grundstruktur des christlichen Bewußtseins darin besteht, das Universum als Geschichte, und wie Schelling erläuternd hinzufügt, als ein moralisches Reich, anzuschauen.

Diese Bestimmung des Christentums ergibt sich zunächst aus seinem

Gegensatz zur Religion des griechischen Altertums. Das bestimmende Gesetz in ihr ist, daß das Unendliche "nur im Endlichen angeschaut" (SW V, 288) wird. Die Anschauung des mythologischen Bewußtseins ist folglich so beschaffen, daß es das Unendliche in das Endliche einbildet. "Die Natur ist allgemein die Sphäre des in-sich selbst-Seyns der Dinge, in der diese, kraft der Einbildung des Unendlichen in ihr Endliches, als Symbole der Ideen zugleich ein von ihrer Bedeutung unabhängiges Leben haben." (SW V, 289) Aufgrund dieser strukturellen Verfassung des mythologischen Bewußtseins sind die griechischen Götter "bleibende unwandelbare Gestalten" (SW V, 288). Die Grundbestimmung der heidnischen Religion ist folglich Präsenz. Die

${ }^{21}$ Schellings Bestimmung der christlichen Religion als einer geschichtlichen Anschauung des Universums nimmt deutlich Bezug auf Schleiermachers einschlägige Bestimmung in den Reden Über die Religion von 1799. Den Ort der Religion in der Struktur des Bewußtseins im Unterschied zu den Bewußtseinsvermögen Denken und Handeln, zu denen die Religion in einem "schneidenden Gegensaz" steht, erblickt Schleiermacher in der Anschauung und dem Gefühl. "Sie [sc. die Religion] begehrt nicht das Universum seiner Natur nach zu bestimmen und zu erklären wie die Metaphysik, sie begehrt nicht aus Kraft der Freiheit und der göttlichen Willkühr des Menschen es fortzubilden und fertig zu machen wie die Moral. Ihr Wesen ist weder Denken noch Handeln, sondern Anschauung und Gefühl. Anschauen will sie das Universum, in seinen eigenen Darstellungen und Handlungen will sie es andächtig belauschen, von seinen unmittelbaren Einflüßen will sie sich in kindlicher Paßivität ergreifen und erfüllen laßen." F Schleiermacher, Über die Religion. Reden an die Gebildeten unter ihren Verächtern (1799), Hrsg v G Meckenstock, Berlin/New York 1999, 50 (Paginierung der Erstausgabe). Schelling selbst hatte bekanntlich zunächst Schleiermachers Reden eher abschätzig beurteilt, brachte aber bereits im Sommer 1801 in einem Brief an August Wilhelm Schlegel seine Verehrung für Schleiermachers Schrift zum Ausdruck (vgl Brief Schellings an A.W. Schlegel vom 3. 7. 1801, in G L Plitt (Hrsg), Aus Schellings Leben. In Briefen Bd. 1. Leipzig 1869, 345). Für Schleiermachers Anschauungsbegriff, dem in der ersten Auflage der Reden für die Bestimmung der Religion eine dominante Stellung zukommt, ist der Darstellungsbegriff leitend. Die religiöse Anschauung unterscheidet sich dadurch sowohl von der intellektuellen Anschauung als auch von der sinnlichen Anschauung, daß sie das Einzelne als Darstellung des Universums anschaut (vgl F Schleiermacher, Über die Religion, 56). Schleiermachers Begriff des Universums resultiert aus einer wechselseitigen Korrektur von Kant und Spinoza. Spinozas Bestimmung der Inhärenz von Unendlichem und Endlichem identifiziert Schleiermacher mit der Kantischen Unterscheidung von Noumenon und Phänomenon. Im Resultat führt dies zu der Bestimmung, daß die Religion in ihrem Kern als ein spezifisches Totalitätsbewußtsein verstanden wird. Zu Schleiermachers Religionstheorie in den Reden siehe $\mathrm{C}$ Seysen, Die Rezeption des Atheismusstreites bei F Schleiermacher, in: K-M Kodalle \& M Ohst (Hrsg), Fichtes Entlassung. Der Atheismusstreit vor 200 Jahren, Würzburg 1999, 175-190; G Meckenstock, Deterministische Ethik und kritische Theologie. Die Auseinandersetzung des frühen Schleiermacher mit Kant und Spinoza 1789-1794, Berlin/New York 1988. Zu Schellings Aufnahme des religionstheoretischen Grundbegriffs von Schleiermachers Reden siehe O Ritschl, Studien zur Geschichte der protestantischen Theologie im 19. Jahrhundert, in ZThK 5 (1895), 486-529. 
Synthesis des mythologischen Bewußtseins verbindet das Unendliche mit der endlichen Form, so daß dieses in unterschiedlichen Gestalten präsent ist.

Von dieser Formation des religiösen Bewußtseins unterscheidet sich das Christentum dadurch, daß es "auf das Unendliche unmittelbar an sich selbst geht". Das Verhältnis von Unendlichem und Endlichem baut sich folglich in der christlichen Religion so auf, daß "das Endliche nicht als Symbol des Unendlichen, zugleich um seiner selbst willen, sondern nur als Allegorie des ersten und in der gänzlichen Unterordnung unter dasselbe gedacht" (ebd.) wird. Auch das christliche Bewußtsein verbindet Unendliches und Endliches. Darin ist es wie das mythologische Bewußtsein Anschauung. Die Synthesis von Unendlichem und Endlichem wird jedoch von dem christlichen Bewußtsein so vorgenommen, daß das Endliche als Darstellung des Unendlichen gewußt wird. Das christliche Bewußtsein ist somit gegenüber dem mythologischen Bewußtsein durch eine veränderte Stellung des Bewußtseins zu seinen inhaltlichen Korrelaten ausgezeichnet. Dem christlichen Bewußtsein sind seine inhaltlichen Bestimmungen nicht als solche relevant, sondern nur dadurch, daß sie Darstellungen des Unendlichen sind. Ein solches Bewußtsein kann als ein Differenzbewußtsein angesprochen werden. Es muß um die Differenz von Endlichem und Unendlichem wissen, damit es Endliches als Darstellung des Unendlichen anschauen kann.

Es ist an dieser Stelle auf zwei Aspekte hinzuweisen, durch die das bisher Ausgeführte seine Begründung erfährt. Einerseits geht Schelling davon aus, daß dem Christentum ein, wie er es nennt, "Abbrechen des Menschen von der Natur" (SW V, 290) zugrunde liegt. Dadurch, daß sich die Freiheit als solche erfaßt, treten Natur und Freiheit in einen Widerstreit, der von Schelling Schicksal genannt wird. Diese Antinomie läßt sich nun zweitens nicht mehr objektiv auflösen, sondern nur noch im Subjekt. Schelling nennt diese Handlung "Mysticismus" und erläutert sie als subjektive Symbolik. "Alle Symbolik fällt ins Subjekt zurück, und die nicht äußerlich, sondern bloß innerlich zu schauende Auflösung des Gegensatzes bleibt daher Mysterium, Geheimniß. Die durch alles hindurchgehende Antinomie des Göttlichen und Natürlichen hebt sich allein durch die subjektive Bestimmung auf, beide auf eine unbegreifliche Weise als eins zu denken" (SW V, 292f.).

Das christlich-religiöse Bewußtsein verbindet Endliches und Unendliches so, daß es das Endliche als Endliches negiert und es dadurch in

\footnotetext{
${ }^{22}$ Vgl auch Ueber das Verhältnis der Naturphilosophie zur Philosophie überhaupt, SW V, 188. Siehe auch Vorlesungen, SW V, 290. 294; Philosophie der Kunst, SW V, 443: "Nur ins Subjekt fällt hier die Einheit des Unendlichen und Endlichen. Aber von diesem inneren Mysticismus kann selbst wieder eine sittliche Person das objektive Symbol seyn, und er kann zur poetischen Anschauung gebracht werden, nicht aber wenn man ihn selbst nur wieder sich subjektiv aussprechen läßt."
} 


\section{Schellings Wesensbestimmung des Christentums}

einen Unendlichkeitshorizont hineinstellt. Damit ist das christliche Bewußtsein von Schelling als ein Zeichenbewußtsein bestimmt, dem seine inhaltlichen Bestimmungen als geschichtlich wandelbare Repräsentationen des Absoluten bewußt sind. Die Präsenz des Absoluten ist für das christliche Bewußtsein nur als Repräsentation zu haben. Demzufolge müssen die inhaltlichen Momente des Bewußtseins mit einer Negation versehen werden. Das Unendliche kann sich dann nur indirekt, eben als Negation an dem Endlichen zur Darstellung bringen, und zwar als Übergang von einer Form zur nächsten. Da dies nur in der Zeit möglich ist, ist die Grundbestimmung des christlichen Bewußtseins die Geschichte. ${ }^{23}$ "[Die] Gestalten nicht bleibend, sondern erscheinend, nicht ewige Naturwesen, sondern historische Gestalten, in denen sich das Göttliche nur vorübergehend offenbaret, und deren flüchtige Erscheinung allein durch den Glauben festgehalten werden kann, niemals aber in eine absolute Gegenwart verwandelt wird" (SW V, 288). Der Grundcharakter der christlichen Anschauung des Universums als Geschichte stellt somit ein Implikat des christlich-religiösen Bewußtseins als einem Zeichenbewußtsein dar. Damit ist Schellings Behauptung, daß im Christentum das Universum als Geschichte angeschaut wird, begründet. Eben weil das christlich-religiöse Bewußtsein das Endliche als Darstellung des Absoluten anschaut, verbindet es auf einer neuen Ebene Natur und Freiheit. Schelling nennt diese Synthese Vorsehung. Sie ist mit dem christlich-religiösen Bewußtsein verbunden und resultiert daraus, daß sich in diesem die Vernunft in der Geschichte selbst reflexiv wird. Da dieses Reflexivwerden der Vernunft nicht von der individuellen Einsicht gelöst werden kann, leitet das Christentum "jene Periode der Vorsehung" (SW $\checkmark$, 290) nur ein.

Faßt man diese Überlegungen zusammen, dann kann man sagen, daß das Christentum nicht nur eine historische Erscheinung ist, sondern in ihm wird sich die Vernunft in der Geschichte selbst reflexiv. Aus diesem Grund ist die "Wissenschaft der Religion in inm von der Geschichte unzertrennlich, ja mit ihr völlig eins" (SW V, 291). Die Vernunft, die sich im Christentum selbst reflexiv wird, ist jedoch nicht durch eine partikulare Perspektive zu erfassen, sondern selbst nur aus einer Vernunftperspektive. Schelling nennt diese Vernunftperspektive "höhere Geschichte" und weist ihr den Status zu, die Bedingung der Möglichkeit der historischen Konstruktion des Christentums zu

\footnotetext{
${ }^{23}$ Bereits Paul Tillich hatte in einer 1910 verfaßten Skizze mit dem Titel Gott und das Absolute bei Schelling auf die von Schelling in den Vorlesungen über die Methode des akademischen Studiums vorgenommene geschichtsphilosophische Begründung des Christentums aufmerksam gemacht. Vgl P Tillich, Gott und das Absolute bei Schelling, in ders, Religion, Kultur, Gesellschaft. Unveröffentlichte Texte aus der deutschen Zeit (19081933), Erster Teil, hrsg. von E Sturm, Berlin/New York 1999, 9-54, bes 45: "Die Konstruktion des Christentums ist eine Konstruktion der Geschichte. Das Christentum eröffnet die dritte Weltepoche, die Periode der Vorsehung."
} 
sein. Mit dieser Bedingung ist nicht nur der Theologiebegriff Schellings erreicht, sondern es dürfte jetzt auch deutlich geworden sein, warum die Theologie die höchste Synthese des historischen und philosophischen Wissens darstellt. Dieser Begriff der Theologie ist jetzt abschließend noch in den Blick zu nehmen.

\section{DIE THEOLOGIE ALS KONSTRUKTION DER IDEE DES CHRISTENTUMS}

Der Theologie als der Wissenschaft von der Religion obliegt die Aufgabe einer historischen Konstruktion des Christentums. Dies kommt der Aufgabe gleich, das Wesen oder die Idee des Christentums zu konstruieren. ${ }^{24}$ Dies kann freilich, soviel dürfte durch die bislang angestellten Überlegungen deutlich geworden sein, nicht im Ausgang von der geschichtlich-empirischen Gestalt des Christentums geschehen. Derartige Wesensbestimmungen des Christentums bleiben nicht nur immer partikular, sie unterschreiten auch das Wesen des Christentums. Vielmehr ist die Idee des Christentums immer eine Konstruktion, die aus einer Verbindung des Besonderen, hier des in sich vielspältigen Christentums, mit dem Allgemeinen hervorgeht. Deshalb ist eine solche Konstruktion, sofern sie den Anspruch erhebt, Wissenschaft zu sein, ohne Philosophie nicht möglich. Schelling bestimmt sie aus diesem Grund als "das wahre Organ der Theologie als Wissenschaft [...], worin die höchsten Ideen von dem göttlichen Wesen, der Natur als dem Werkzeug und der Geschichte als der Offenbarung Gottes objektiv werden" (SW V, 299). Mit Schellings Wesensbestimmung des Christentums ist eine Kritik an der Theologie seiner Zeit verbunden, die eine unmittelbare Konsequenz seines Theologiebegriffs darstellt. Ich beginne mit Schellings Kritik an der zeitgenössischen Theologie und gehe dann auf die von Schelling selbst vorgenommene Begründung der Theologie als Wissenschaft ein.

Nach Schellings Urteil sind in der Theologie seiner Zeit "sämmtliche[..] Lehren dieser Wissenschaft [...] empirisch verstanden und als solche sowohl behauptet als bestritten worden" (SW V, 296). In dieses Urteil über seine zeitgenössische Theologie bezieht Schelling sowohl die Aufklärungstheologie in ihren beiden Spielarten des Rationalismus und des Supranaturalismus als

\footnotetext{
${ }^{24}$ Mit diesem Programm schreibt Schelling, was angesichts seiner Kritik an der Aufklärungstheologie nicht übersehen werden sollte, das Anliegen der Aufklärung mit anderen begrifflichen Mitteln fort. Zu den Debatten um das Wesen des Christentums siehe M Schröder, Die kritische Identität neuzeitlichen Christentums. Schleiermachers Wesensbestimmung der christlichen Religion, Tübingen 1996.
} 


\section{Schellings Wesensbestimmung des Christentums}

auch die Kantische Ethikotheologie mit ein. ${ }^{25}$ Im Kern zielt Schellings Kritik auf ein Begründungsdefizit dieser Theologien. Es besteht darin, daß sich die Behauptungen der Theologen über das Christentum und seine Geltung auf einer empirisch-historischen Ebene weder begründen noch widerlegen lassen. Um die Geltung des Christentums zu begründen, müssen sie die Idee des Christentums in Anspruch nehmen. Aber eben diese wird von der unterschiedlichen Theologien nicht begriffen, sondern selbst empirisch gedeutet. "Die christlichen Religionslehrer können keine ihrer historischen Behauptungen rechtfertigen, ohne zuvor die höhere Ansicht der Geschichte selbst, welche durch die Philosophie wie durch das Christenthum vorgeschrieben ist, zu der ihrigen gemacht zu haben." (SW V, 297) In den unterschiedlichen Theologien seiner Zeit tritt in den Augen Schellings das empirische Christentum an die Stelle von dessen Idee. Dieses Begründungsdefizit der Theologien versucht Schelling in den Vorlesungen über die Methode des akademischen Studiums an dem Rationalismus, dem Supranaturalismus sowie an der Kantischen Ethikotheologie nachzuweisen.

Was zunächst die rationalistischen Aufklärungstheologien betrifft, so sieht Schelling in diesen infolge der von ihr betriebenen Dogmenkritik das Urchristentum an die Stelle der für den Altprotestantismus inspirierten Heiligen Schrift als normativer Instanz der Theologie treten. "Zu den Operationen der neuern Aufklärerei, welche in Bezug auf das Christenthum eher Ausklärerei heißen könnte, gehört allerdings auch das Vorgeben, es, wie man sagt, auf seinen ursprünglichen Sinn, seine erste Einfachheit zurückzuführen, in welcher Gestalt sie es auch das Urchristenthum nennen." (SW V, 300) Die Auszeichnung des Urchristentums als in geltungstheoretischer Hinsicht normativer Instanz verdankt sich jedoch selbst einerseits partikularen Interessen und wird andererseits der geschichtlichen Vielschichtigkeit des sogenannten Urchristentums nicht gerecht. Schellings Kritik an dem begründungstheoretischen Verfahren der Aufklärungstheologie zielt genau auf diesen Aspekt, daß das Urchristentum keine einheitliche Größe darstellt. Angesichts dieses Umstands kommt aber die Erhebung des Urchristentums zur normativen Instanz einem Akt der Willkür gleich, der die Komplexität des Urchristentums nach der Maßgabe von gegenwärtigen Interessen reduzieren muß. "Schon in dem Geiste des Heidenbekehrers Paulus ist das Christenthum etwas anderes geworden, als es in dem des ersten Stifters war: nicht bei der einzelnen Zeit sollen wir stehen bleiben, die nur willkürlich angenommen werden kann, sondern seine ganze Geschichte

\footnotetext{
${ }^{25}$ Zur genaueren Bestimmung der mit den Stichworten "Rationalismus" und "Supranaturalismus" markierten Positionen siehe v Vf, Art: Naturalismus II III, in Religion in Geschichte und Gegenwart Bd. 6, Tübingen ${ }^{4} 2003$, Sp 111-113.
} 
und die Welt, die es geschaffen, vor Augen haben." (ebd.) ${ }^{26}$ Aufgrund dieser begründungstheoretischen Schwächen kann die Geltung des Christentums nicht durch einen Rekurs auf eine angeblich normative Urgestalt des Christentums begründet werden.

Im Unterschied zur rationalistischen Aufklärungstheologie hält zwar der Supranaturalismus an der Heiligen Schrift als normativer Quelle der Theologie fest, aber da er unter dem Eindruck der historischen Bibelkritik das Insprirationsdogma faktisch auf die Glaubwürdigkeit der ersten Zeugen zurücknimmt, bleibt auch er auf einer historisch-empirischen Ebene stehen. "Der Glaube an seine Göttlichkeit [sc. des Christentums] wurde auf empirischhistorische Argumente gebaut, das Wunder der Offenbarung in einem sehr

\footnotetext{
${ }^{26}$ Die von Schelling geltend gemachte Differenz von Jesus und Paulus gegenüber einer vermeintlichen Homogenität des Urchristentums sowie die sich daran anschließende methodische Maxime, die gesamte Geschichte des Christentums in die Bestimmung des Wesens des Christentums einzubeziehen, ist der Sache nach von Ernst Troeltsch in seinen methodologischen Überlegungen zum Problem der Wesensbestimmung des Christentums aufgenommen und weitergeführt worden. Vgl E Troeltsch, Was heisst "Wesen des Christentums"?, in ders, Gesammelte Schriften II. Zur religiösen Lage, Religionsphilosophie und Ethik, Tübingen ${ }^{2} 1922$, 386-451. Schellings in den Vorlesungen über die Methode des akademischen Studiums vorgenommene Kritik an der Aufklärungstheologie und deren Handhabung der historischen Kritik setzt diese voraus. Auch seine Bemerkung, daß es die "Zurückweisung auf den Buchstaben einiger Bücher [...] nothwendig [machte], daß die ganze Wissenschaft [sc der Theologie] sich in Philologie und Auslegekunst verwandelte, wodurch sie eine gänzlich profane Scienz geworden ist” (SW V, 302), ist differenziert zu lesen. Sie intendiert keine generelle Verabschiedung der historisch-kritischen Methode zugunsten einer dogmatischen Betrachtung der Bibel, wohl aber eine Kritik an dem einseitigen Gebrauch der grammatischen Methode der Bibelauslegung. Bereits der junge Schelling kritisierte in einer Skizze aus dem Jahre 1793/94, die unter dem Titel Entwurf einer Vorrede überliefert ist, die seit Ernesti geübte "grammatische Interpretation" als ungeschichtliche Abstraktion. "Man betrachtete nur gar zu oft die heil. Urkunden als Schriften, die plötzlich vom Himmel gefallen wären, die man aus allem Zusammenhang herausnehmen und als ganz isolirte Denkmale betrachten müsse, die unabhängig von den Vorstellungen, den Bedürfnissen und allen Umständen derjenigen Zeit, in der sie entstanden, nur auf ein in entfernten Jahrhunderten erst vollkommen auszubildendes System berechnet wären, in die man also auch alle mögliche Weisheit, ohne Rücksicht auf die Empfänglichkeit derjenigen Menschen, denen sie zunächst bestimmt wären, hineintragen dürfte, wenn sie nur zuvor durch das hergebrachte System geheiligt wäre, das denn doch wieder nur aus jenen Schriften geschöpft seyn sollte." (F W J Schelling, [Entwurf einer Vorrede], in L Pareyson (Hrsg), Schellingiana Rariora, Torino 1977, 37-43, hier 41) Aus diesem Grund plädierte Schelling in seiner Skizze für eine Erweiterung der grammatischen Interpretation durch eine historische Interpretation. "Historische Interpretation im weiteren Sinne befasst demnach nicht nur grammatische, sondern auch historische Interpretation, im engeren Sinn dieses Worts. Jene geht blos auf die Bedeutung der Worte, auf ihre verschiedenen Wendungen, Formen und Construktionen, diese nimmt die Belege aus der Geschichte überhaupt, insbesondere aber aus der Geschichte der Zeit, aus der die Urkunde, welche ausgelegt werden soll, herstammt, aus dem Geist, den Begriffen, den Vorstellungs- und Darstellungsarten, die jener Zeit eigenthümlich sind." (ebd, 43) Diese Fragestellung führt aber auf das Problem von Kriterien, nach welchen "die Form einer gewissen Behauptung von der Behauptung selbst, die Form einer Ge[schichte von der Geschichte selbst]" (ebd) unterschieden werden kann. Schellings wissenschaftstheoretische Begründung des Theologiebegriffs in den Vorlesungen über die Methode des akademischen Studiums kann als eine Antwort auf diese Problemstellung gelten.
} 


\section{Schellings Wesensbestimmung des Christentums}

handgreiflichen Cirkel durch andere Wunder bewiesen" (SW V, 302). ${ }^{27}$ Im Resultat identifiziert auch der theologische Supranaturalismus der Aufklärungstheologie das Wesen des Christentums mit der Bibel und damit mit einer bestimmten historischen Gestalt. Aus diesem Grund bleibt die von inm vorgenommene Begründung des Christentums nicht nur partikular, sondern auch willkürlich.

Die von Kant vorgenommene Geltungsbegründung der Religion im Kontext des Anwendungsproblems der reinen praktischen Vernunft auf endlich-sinnliche Vernunftwesen setzt schließlich nicht auf einer empirischhistorischen Ebene an, sondern auf der Ebene der Vernunftreligion. Dadurch tritt zwar bei Kant die Vernunft an die Stelle des empirischen Christentums, aber das Verhältnis von Vernunftreligion und empirischem Christentum bestimmt Kant in der Lesart Schellings so, daß dieses lediglich als Vehikel von jenem fungiert. Deshalb zielt Kants begründungstheoretische Rekonstruktion des Christentums in den Augen Schellings tendenziell darauf, daß "das Positive und Historische aus dem Christenthum gänzlich" entfernt und "zur reinen Vernunftreligion" (SW V, 299) geläutert wird. Während in dem theologischen Rationalismus und in seinem Seitenstück, dem Supranaturalismus, letztlich das empirische Christentum an die Stelle von dessen Idee tritt, tritt bei Kant die Vernunft an die Stelle des empirischen Christentums.

Aufgrund dieses reduktiven Verfahrens erhebt sich auch Kant, so Schellings Argument, nicht zur Idee des Christentums. Da aber Kant die religiösen Vorstellungen des Christentums lediglich als Vehikel der Vernunftreligion zu begreifen vermag, bleibt auch die Moralreligion auf die empirische Sittlichkeit bezogen und somit letztlich empirisch. "In der Kantischen Läuterung ist auch keineswegs ein speculativer, sondern ein moralischer Sinn jener Lehren beabsichtigt, wodurch der empirische Standpunkt im Grunde nicht verlassen, auch die Wahrheit derselben nicht an sich, sondern allein in der subjektiven Beziehung möglicher Motive der Sittlichkeit angenommen wird" (ebd).

Die von Schelling in den Vorlesungen über die Methode des akademischen Studiums vorgenommene Begründung der Theologie als Wissenschaft versteht sich als Alternative zu den in begründungstheoretischer

\footnotetext{
${ }^{27}$ Siehe hierzu G C Storr, Lehrbuch der Christlichen Dogmatik ins Deutsche übersetzt, mit Erläuterungen aus andern, vornehmlich des Verfassens eigenen, Schriften und mit Zusätzen aus der theologischen Literatur seit dem Jahre 1793 versehen von M C C Flatt, Tübingen 1803. Storr ersetzt in seinem Lehrbuch der Christlichen Dogmatik die Inspirationslehre durch historische Untersuchungen, wendet die historische Methode jedoch gegen die Neologie. Im ersten Buch seiner Dogmatik behandelt er die Lehre von der Schrift, deren Geltungsanspruch er durch philologische Untersuchungen sowie des Rekurs auf die Glaubwürdigkeit der Apostel abzustützen versucht. Das von Storr aufgeworfene Problem der Vermittlung von Geschichte und Geltung nimmt Schelling in den Vorlesungen über die Methode des akademischen Studiums auf und beantwortet es durch eine geschichtsphilosophische Bestimmung der Idee des Christentums.
} 
Hinsicht defizitär bleibenden Versuchen der Aufklärungstheologie in ihren unterschiedlichen Spielarten. Diese kommt ihrer Unterschiedlichkeit zum Trotz darin überein, daß Wesen des Christentums auf empirische Weise zu bestimmen. Durch seinen identitätsphilosophischen Ansatz, der sowohl an dem Zusammenhang von Besonderem und Allgemeinem als auch an deren Differenz orientiert ist, überschreitet Schelling diese empirischen Verfahrensweisen. Im methodischen Rahmen der identitätsphilosophischen Wissenschaftssystematik obliegt der Theologie als Wissenschaft die Aufgabe, die Idee des Christentums zu konstruieren. Darin wird die Theologie zur Geschichtsphilosophie. Sie expliziert die Logik, welche Geschichte erst hervorbringt. Die Einlösung dieser Aufgabe kommt für Schelling der Aufgabe gleich, die Trinitätslehre zu explizieren. Diese ist nämlich die zusammenfassende Darstellung der Idee des Christentums. Folglich stellt die Idee der Dreieinigkeit den Leitbegriff einer Theologie als Wissenschaft von der christlichen Religion dar. "Versöhnung des von Gott abgefallenen Endlichen durch seine eigne Geburt in die Endlichkeit ist der erste Gedanke des Christenthums und die Vollendung seiner ganzen Ansicht des Universums und der Geschichte desselben in der Idee der Dreieinigkeit, welche eben deßwegen in inm schlechthin nothwendig ist" (SW V, 294). Die Trinitätslehre kann aus dem Grund als ein zusammenfassender Ausdruck der Idee des Christentums gelten, da in ihr die Momente in ihrem inneren Zusammenhang festgehalten sind, aus denen der Grundcharakter des Christentums erst resultiert, nämlich Anschauung des Universums als Geschichte zu sein. In der Idee der Dreieinigkeit thematisiert das christlich-religiöse Bewußtsein folglich die Regel, der alle christlichen Anschauungen des Universums unterliegen. ${ }^{28}$ Diese besteht, wie oben dargestellt, darin, daß das Endliche als eine Darstellung des Unendlichen angeschaut wird. Somit kommt in der Trinitätslehre das Verfahren der philosophischen Konstruktion selbst zur Darstellung. Deshalb hat diese Lehre nicht nur eine Beziehung auf die Geschichte, wie Schelling kritisch gegenüber Lessing anmerkt, sondern die "Idee der Dreieinigkeit" (SW V, 297) ist auch "von ganz philosophischem Gehalt" (SW V, 431). Sie verliert daher zwangsläufig jeden Sinn, wird sie "nicht spekulativ aufgefaßt" (SW V, 297).

\footnotetext{
${ }^{28}$ Auch in den sich um 1800 herausbildenden spekulativen Theologien, die, wie etwa bei Carl Daub, an die methodischen Grundlagen von Schellings Identitätsphilosophie anschließen, wird die Trinitätslehre zum Inbegriff einer Theologie als Wissenschaft. Bei dem frühen Daub ist die spekulativ rekonstruierte Trinitätslehre jedoch nicht Ausdruck der Logik, welche dem christlich-religiösen Bewußtsein zugrunde liegt, wie bei Schelling, sondern noch stark an das kirchliche Dogma angelehnt. Vgl C Daub, Einleitung in das Studium der christlichen Dogmatik aus dem Standpunkte der Religion, Heidelberg 1810, 297ff. Siehe hierzu v Vf, Gott und die Geschichte. Aspekte der Schelling-Rezeption in der evangelischen Theologie, in: Kritische und absolute Transzendenz. Religionsphilosophie und Philosophische Theologie bei Kant und Schelling, hrsg. V C Danz \& R Langthaler, München/Freiburg 2006, 243-269.
} 
Das Christentum überwindet den Gegensatz von Besonderem und Allgemeinem dadurch, daß es das Besondere als Darstellung des Allgemeinen anschaut. Das Zustandekommen dieses Darstellungsverhältnisses findet seinen Ausdruck in der Idee des Christentums, nämlich in den Vorstellungen der Menschwerdung und des Todes Gottes. Schellings Behauptung ist also nicht die, daß das Absolute "in einem bestimmten Moment der Zeit menschliche Natur angenommen habe". Hierbei sei, so Schelling, "schlechterdings nichts zu denken" (SW V, 298), da unter den Prämissen der Identitätsphilosophie ein Übergang vom Unendlichen zum Endlichen nicht statt haben kann. ${ }^{29}$ Der Gehalt der Idee der Menschwerdung Gottes liegt vielmehr darin, daß ein solcher Zusammenhang zwischen dem Endlichen und dem Unendlichen aufgebaut wird, in dem das Unendliche dadurch am Endlichen zur Darstellung kommt, daß sich dieses als solches negiert. Allein dadurch kann das Endliche zu einer Darstellung des Unendlichen werden. Diesen Verweisungszusammenhang zwischen Endlichem und Absolutem hat Schelling in seinem Begriff des Geistes festgehalten. Als das "ideale Princip" (SW V, 292) ${ }^{30}$ beinhalte er ein solches Verhältnis, in dem das Endliche sowohl in Identität als auch in Differenz zum Absoluten steht. Eben dies ist der Inhalt der christlichen Religion. Dadurch überwindet sie nicht nur den Widerstreit zwischen Endlichem und Unendlichem, sondern objektiviert auch den inneren Typus der Philosophie. Die Theologie als besondere Wissenschaft stellt somit nicht den Indifferenzpunkt als solchen dar, wie Schleiermacher meinte, sondern allein das Verfahren der Philosophie.

Auf dieser Bestimmung der Idee des Christentums fußt Schellings im Stile unversöhnlicher Polemik vorgetragene Kritik an der zeitgenössischen

\footnotetext{
${ }^{29}$ Dieser Gedanke Schellings wurde von David Friedrich Strauß in seiner spekulativen Rekonstruktion der Christologie in der Schlußabhandlung seines Leben Jesu aufgenommen und zu seiner Gattungschristologie weitergeführt. Vgl. D.F. Strauß, Das Leben Jesu. Kritisch bearbeitet, Zweiter Bd., Tübingen ${ }^{2}$ 1837, 734: "Schon Kant hatte gesagt, das gute Princip sei nicht bloß zu einer gewissen Zeit, sondern vom Ursprung des menschlichen Geschlechts an unsichtbarerweise vom Himmel in die Menschheit herabgekommen, und Schelling stellt den Satz auf: die Menschwerdung Gottes ist eine Menschwerdung von Ewigkeit. Aber während der erstere unter jenem Ausdruck nur die moralische Anlage verstanden hatte, welche mit jenem Ideal und ihrem Sollen von jeher dem Menschen eingepflanzt sei: verstand der letztere unter dem menschgewordenen Sohn Gottes das Endliche selbst, wie es im Menschen zum Bewußtsein kommt, und in seinem Unterschied von dem Unendlichen, mit dem es doch Eins ist, als ein leidender und den Verhältnissen der Zeit unterworfener Gott erscheint."

${ }^{30}$ Vorlesungen, SW V, 292: "Die erste Idee des Christenthums ist daher nothwendig der Menschgewordene Gott, Christus als Gipfel und Ende der alten Götterwelt. Auch er verendlicht in sich das Göttliche, aber er zieht nicht die Menschheit in ihrer Hoheit, sondern in ihrer Niedrigkeit an, und steht als eine von Ewigkeit zwar beschlossene, aber in der Zeit vergängliche Erscheinung da, als Grenze der beiden Welten; er selbst geht zurück ins Unsichtbare, und verheißt statt seiner nicht das ins Endliche kommende, im Endlichen bleibende Princip, sondern den Geist, das ideale Princip, welches vielmehr das Endliche zum Unendlichen zurückführt und als solches das Licht der neuen Welt ist."
} 
Theologie. Sie zielt, wie deutlich geworden sein dürfte, im Kern auf eine Verwechslung von Idee und Erscheinung. In den unterschiedlichen Spielarten der Theologie seiner Zeit trete die empirisch historische Erscheinung des Christentums an die Stelle der Idee. Damit ist die Konsequenz verbunden, daß derartige Formen von Theologie nicht nur nicht dem Wesen des Christentums selbst gerecht werden, sondern auch, daß sie die besonderen Formen des geschichtlichen Christentums absolut setzen. Die Theologie schreibt auf diese Weise die Entzweiung fort, welche zwar das Signum der modernen Kultur ist, die aber durch das Christentum im Prinzip schon überwunden ist. Für Schelling beinhaltet die Idee des Christentums eine Transformationslogik, nach der dieses nur dann seiner Idee entspricht, wenn es seine besonderen Formen beständig überschreitet. Er vertritt damit die Auffassung, daß eine geltungstheoretische Wesensbestimmung des Christentums weder dem empirischen Christentum als solchem entnommen werden kann, noch einer angeblichen Urgestalt des Christentums oder den biblischen Schriften, sondern allein der Idee des Christentums. Diese liegt aber nicht einfach vor, sondern sie verdankt sich selbst erst der philosophischen Konstruktion.

\section{Quellenforschung}

Bachmann, M 1994. Die paradoxale Struktur des Absoluten in Schellings Identitätssystem. PhJb 101, 76-97.

Becker, W 1966. Über Schellings Konstruktion des Christentums, in Henrich, D \& Wagner, H (Hrsg), Subjektivität und Metaphysik, 1-20. Festschrift für Wolfgang Cramer. Frankfurt: Klostermann Verlag.

Danz, C 2002. Vernunft und Religion: Überlegungen zu Schellings Christentumsdeutung in seinen Journal-Aufsätzen, in Vieweg, K (Hrsg), Gegen das unphilosophische Unwesen. 197-209. Würzburg: Königshausen \& Neumann Verlag,

Danz, C 2003. Art: Naturalismus II III, in Religion in Geschichte und Gegenwart, Bd. 6, Sp. 111-113. 4 Auflage. Tübingen: Mohr.

Danz, C 2006. Gott und die Geschichte: Aspekte der Schelling-Rezeption in der evangelischen Theologie, in von Danz, C \& Langthaler, R (Hrsg). Kritische und absolute Transzendenz: Religionsphilosophie und Philosophische Theologie bei Kant und Schelling, 243-269, München: Alber Verlag.

Daub, C 1810. Einleitung in das Studium der christlichen Dogmatik aus dem Standpunkte der Religion. Heidelberg: Mohr \& Zimmer Verlag.

Dierken, J 1992. Das Absolute und die Wissenschaften: Zur Architektonik des Wissens bei Schelling und Schleiermacher. PhJb 99, 307-328.

Düsing, K 1988. Die erste Gestalt der Metaphysik des Absoluten bei Schelling (Vorlesung und Schriften 1801-1802), in ders (Hrsg), Schellings und Hegels erste absolute Metaphysik (1801-1802). Zusammenfassende Vorlesungsnachschriften von I P V Troxler, 133-156. Köln: Dinter Verlag. 


\section{Schellings Wesensbestimmung des Christentums}

Hennigfeld, J 1989. Einheit und Vielheit als grundlegendes Problem in Schellings Systementwürfen. AZP 14, 1-15.

Hirsch, E 1952. Geschichte der neuern evangelischen Theologie im Zusammenhang mit den allgemeinen Bewegungen des europäischen Denkens, Bd. 4. Gütersloh: Bertelsmann Verlag.

Jaeschke, W 1986. Die Vernunft in der Religion: Studien zur Grundlegung der Religionsphilosophie Hegels. Stuttgart-Bad Cannstatt: Frommann-Holzboog Verlag.

Meckenstock, G 1988. Deterministische Ethik und kritische Theologie: Die Auseinandersetzung des frühen Schleiermacher mit Kant und Spinoza 17891794. Berlin: De Gruyter Verlag.

Plitt, G L 2003. Aus Schellings Leben (1869), in Briefen Bd. 1. Leipzig: Olms Verlag.

Ritschl, O 1895. Studien zur Geschichte der protestantischen Theologie im 19. Jahrhundert. ZThK 5, 486-529.

Rosenau, H 1985. Die Differenz im christologischen Denken Schellings. Frankfurt: Lang.

Schelling, F W J 1856-61. Sämmtliche Werke, Bd. I-XIV, hrsg. von K F A Schelling. Stuttgart: Cotta.

Schelling, F W J 1977. [Entwurf einer Vorrede], in Pareyson, L (Hrsg), Schellingiana Rariora, 37-43. Torino: Bottega d'Erasmo Verlag.

Schleiermacher, F 1863. Rezension F W J Schelling, Vorlesungen über die Methode des akademischen Studiums, 1803, in Jenaische Litteraturzeitung, Bd. I, No. 96. 97 (1804), 137-151, wiederabgedruckt in Dilthey, W (Hrsg), Aus Schleiermacher's Leben, in Briefen. 4. Bd, 579-593. Berlin: Georg Reimer.

Schleiermacher, F 1999. Über die Religion: Reden an die Gebildeten unter ihren Verächtern (1799). Hrsg von Meckenstock, G. Berlin: De Gruyter Verlag.

Schröder, M 1996. Die kritische Identität neuzeitlichen Christentums: Schleiermachers Wesensbestimmung der christlichen Religion. Tübingen: Mohr Verlag.

Seysen, C 1999. Die Rezeption des Atheismusstreites bei F Schleiermacher, in Kodalle, K-M, \& Ohst, M (Hrsg), Fichtes Entlassung: Der Atheismusstreit vor 200 Jahren, 175-190. Würzburg: Königshausen \& Neumann Verlag.

Storr, G C 1803. Lehrbuch der Christlichen Dogmatik ins Deutsche übersetzt, mit Erläuterungen aus andern, vornehmlich des Verfassens eigenen, Schriften und mit Zusätzen aus der theologischen Literatur seit dem Jahre 1793 versehen von M C C Flatt. Tübingen: Metzler Verlag.

Strauß, D F 1837. Das Leben Jesu: Kritisch bearbeitet, Zweiter Bd. 2. Auflage. Tübingen: Saur.

Tillich, P 1999. Gott und das Absolute bei Schelling, in ders, Religion, Kultur, Gesellschaft: Unveröffentlichte Texte aus der deutschen Zeit (1908-1933), 954. Erster Teil, hrsg. von E Sturm. Berlin: de Gruyter Verlag.

Troeltsch, E 1922. Was heisst "Wesen des Christentums"?, in ders, Gesammelte Schriften II. Zur religiösen Lage, Religionsphilosophie und Ethik, 386-451. 2. Auflage. Tübingen: Mohr. 
Von Zantwijk, T 2002. Ist Anthropologie als Wissenschaft möglich? Der "Mensch", in Schmids "enzyklopädischer Topik" und in Schellings "philosophischer Konstruktion der Wissenschaften", in Jantzen, J \& Österreich, P L (Hrsg), Schellings philosophische Anthropologie, 110-154. Stuttgart-Bad Cannstatt: Frommann-Holzboog Verlag.

Ziche, P 2001. Wissenschaft vom Menschen und Philosophie der Natur:

Anthropologie zwischen Physiologie und Naturwissenschaften, in Breitbach, O \& Ziche, P (Hrsg), Naturwissenschaften um 1800: Wissenschaftskultur in Weimar/Jena. Weimar: Böhlau Verlag.

Ziche, P 2002. Die "eine Wissenschaft der Philosophie" und die "verschiedenen philosophischen Wissenschaften": Wissenschafts-systematik und die Darstellung des Absoluten in Ueber das Verhältnis der Naturphilosophie zur Philosophie überhaupt, in Vieweg, K (Hrsg), Gegen das "unphilosophische Unwesen": Das Kritische Journal der Philosophie von Schelling und Hegel, 211-222. Würzburg: Königshausen \& Neumann Verlag. 